\title{
Differentielle Zellteilungen und Polyploidie bei der Schuppenbildung der Mehlmotte Ephestia kühniella $Z$
}

\author{
Von Karl Henke und Heinz-Joachim Pohley \\ Aus dem Zoologischen Institut der Universität Göttingen \\ (Z. Naturforschg. 7 b, 65-79 [1952]; eingegangen am 6. Dezember 1951).
}

Die Zellen der Hinterflügelanlage von Ephestia durchlaufen während der Mitosenperioden der Vorpuppen- und Puppenzeit 5 differentielle Zellteilungsschritte DT $1-5$, von denen der erste neu ermittelt wird (Abb. 1). DT 1 und 2 führen jeweils zur Bildung von 4 verschiedenwertigen Stammzellen (StZ), die nach ihrer Deszendenz als Schuppenepithel-StZ bezeichnet werden. Sie zerfallen mit der DT 3 gleichermaßen in je eine Schuppen-StZ I, die mit den DT 4 und 5 die Zellen des Schuppenapparates liefert, und eine Epithel-StZ, aus welcher ein Epithelzellverband hervorgeht. Durch Behandlung mit Röntgenstrahlen kann die im Zellbild nicht kenntliche DT 3 experimentell erfaßt werden, da die Epithel-StZ und ihre Nachkommen weitgehend resistent sind, ihre Schwesterzellen dagegen, die Schuppen-StZ I, mit ihren Abkömmlingen sowie die gemeinsamen Vorfahrenzellen hochempfindlich.

- Die Verschiedenwertigkeit der Schuppenepithel-StZ, die mit den DT 1 und 2 festgelegt wird, prägt sich stets in einer verschiedenen Größe der aus ihnen hervorgehenden Schuppen aus. Bei Überschreitung bestimmter Grenzen treten Unterschiede der Polyploidiestufen der Schuppenbildungszellen hinzu, welche neuerdings außer an den Kerngrößen auch an den Anzahlen der Chromozentren in den Ruhekernen der Bildungszellen bestimmt werden. Es wird geschlossen, daß die Polyploidiestufen 'plasmatisch bedingt sind, doch wirken sie auch ihrerseits auf die Schuppengröße.

Die verschiedenen Polyploidiestufen der Schuppenbildner sind nach dem Kompensationsprinzip jeweils mit verschiedenen Teilungspotenzen der zugehörigen Epithel-StZ verknüpft, derart, daß die Anzahl der Endomitoseschritte, welche die Polyploidiestufe der Schuppe einstellt, mit der Anzahl der von der Epithel-StZ durchlaufenen Vermehrungsteilungsschritte eine konstante Summe bildet. Auf Grund dieses Prinzips wird nach Bestimmung des Häufigkeitsverhältnisses zwischen Schuppen- und Epithelzellen in verschiedenen Flügelregionen ein bisher nicht bekannter Schuppengrößentyp mit der Kernstufe $4 \mathrm{n}$ vorausgesagt und gefunden.

Die nach Strahlenwirkung häufig auftretende somatische Mutation dunkle Schuppen erscheint bei Behandlung der Tiere zu verschiedenen Zeiten nach der letzten Raupenhäutung aber vor Einsetzen der Vorpuppenmitosenperiode in mutanten Schuppennestern, deren Gliedanzahl mit fortschreitendem Behandlungsalter der Tiere abnimmt. Hieraus folgt, daß die mutationsfähige Anlage in den Blastemzellen während der fraglichen Zeit fortschreitend in eine zunehmende Anzahl selbständig mutationsfähiger Einheiten zerlegt wird, die in der anschließenden Mitosenperiode auf die Abkömmlinge der einzelnen Zellen verteilt werden. Die Annahme, daß diese Verteilung sich nicht streng nach der Entstehungsfolge der Mutationseinheiten richtet, sondern mehr oder weniger zufallsmäßig erfolgt, bietet eine Möglichkeit der Erklärung für die Inkongruenz zwischen genealogischen Schuppenverbänden und Mutantennestern neben zwei anderen, die geprüft werden können.

$I^{\prime}$ n der Metamorphose der Schmetterlinge erfährt das Epithel der Körperdecke tiefgreifende Änderungen. Eine von ihnen besteht in der Herausbildung des Schuppenkleides, die mit einer Folge von differentiellen Zellteilungen verknüpft ist. Die Untersuchungen, von denen hier berichtet wird, erweitern und vertiefen unsere bisherigen Kenntnisse ${ }^{1}$ von den Teilungsvorgängen in der Entwicklung des beschuppten Falterepithels. Wir danken Professor $\mathrm{H}$ a n s

1 K. H e n k e, Naturwiss. 34, 149 [1947].
B a u e r für kritische Diskussion der Befunde, Frau Christine Lipp für ihre Hilfe bei den zytologischen Untersuchungen sowie Frau Waltraud Qu erner, die uns bei den Kernmessungen unterstützte.

Für den Flügel der Mehlmotte wurden bisher vier differentielle Teilungsschritte beobachtet oder erschlossen; nach den hier geschilderten neuen Untersuchungen geht ihnen teilweise noch ein weiterer voraus (Abb. 1). 
Die beiden letzten, während der Mitosenperiode des Puppenstadiums ablaufenden Schritte (DT 4 und 5) sind morphologisch nachweisbar ${ }^{2}$. Sie lassen aus einer Schuppenstammzelle I. Ordnung (SchStZ I) die Bildungszellein von Schuppe und Balg sowie eine in den meisten Fällen degenerierende Zelle hervorgehen. Diese letzte kann jetzt nach Untersuchungen ${ }^{3}$ an metamorphosierenden Trichopteren und an Raupen der Wachsmotte aus Analogiegründen als Sinnesstammzelle bezeichnet werden, da eine im Entwicklungsgang verschiedener Sinnesorgane an entsprechender Stelle auftretende Zelle bald eine Gruppe von vier Sinneszellen hervorbringt, bald selbst unmittelbar in eine einzelne derartige Zelle übergeht. Die verschiedenen Sinnesorgane des Schmetterlingsflügels ent- epithelstammzellen (SchEStZ) durchlaufenen, jetzt als DT 3 bezeichneten differentiellen Zellteilung, bei der jeweils eine SchStZ I und eine Epithelstammzelle (EStZ) entsteht, welche in den fraglichen Flügelgebieten in drei Teilungsschritten 8 Epithelzellen liefert.

Die relativ kleinen Schuppen solcher Flügelgebiete entsprechen dem niedersten der drei bisher bekannten Schuppengrößentypen, den Tiefenschuppen, die, nach dem Volumverhältnis ihrer Bildungszellkerne zu den Epithelkernen zu schließen, aus oktoploiden Zellen hervorgehen ${ }^{6}$. In Flügelgebieten, welche zwischen Tiefenschuppen Vertreter der beiden höheren Größentypen, der Mittel- und Deckschuppen mit den Kernstufen $16 \mathrm{n}$ und $32 \mathrm{n}$ führen, ist das SchE-Verhältnis anders. Auf der Unterseite des

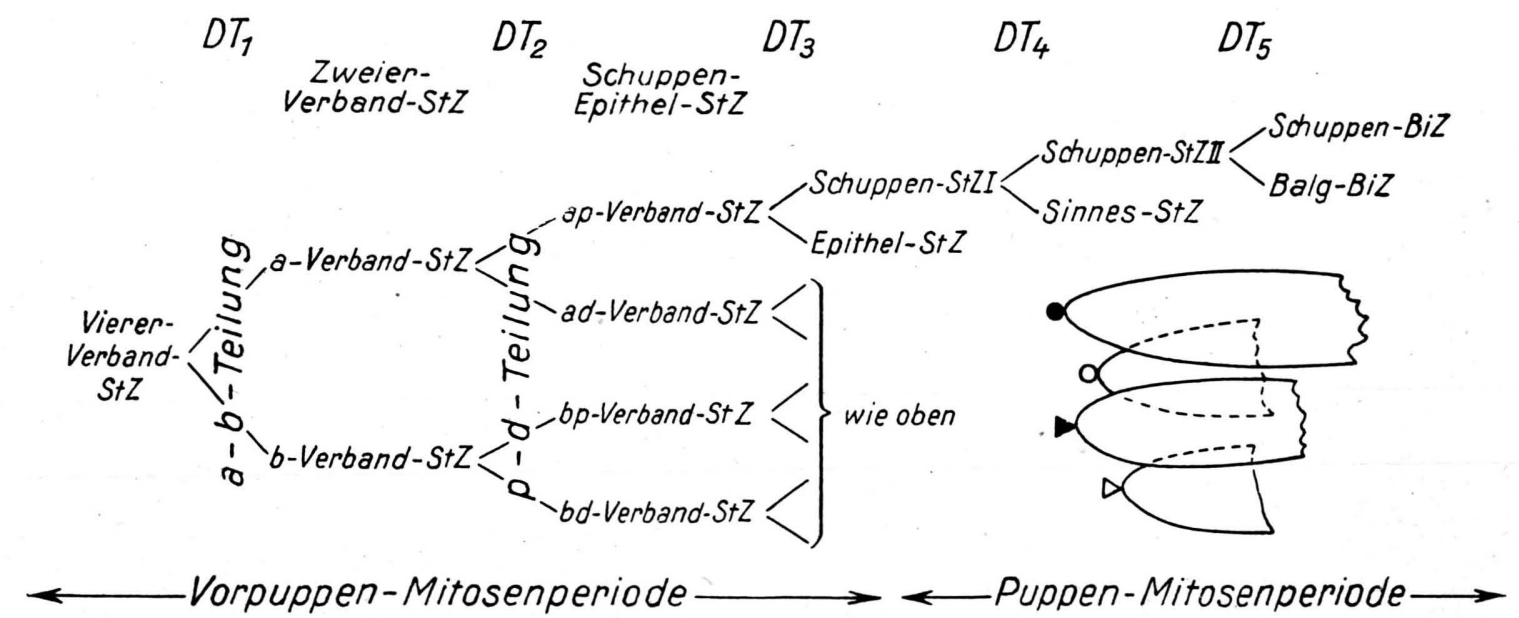

Abb. 1. Übersicht der 5 differentiellen Teilungsschritte (DT 1-5) bei der Metamorphose des Flügelepithels der Mehlmotte. StZ Stammzellen, BiZ Bildungszellen. Rechts die Schuppen eines Viererverbandes aus einem 5,5-Gebiet des Hinterflügels. 200/1.

stehen vermutlich nach demselben weitverbreiteten Plan, und das Auftreten der clegenerierenden Zelle in der Genealogie der gewöhnlichen Schuppenapparate der Schmetterlinge ist ein Ausdruck für die Bildungsgleichheit dieser letzten mit Sinnesorganen.

Die DT 2 und 3 wurden zunächst aus Untersuchungen über die drei von $\mathrm{K} \ddot{\mathrm{uhn}}$ und $\mathrm{Hen} \mathrm{ke}^{4}$ aufgestellten Größentypen der Schuppen und ihre Verteilung im Epithel der verschiedenen Flügelflächen der Mehlmotte erschlossen ${ }^{5}$. Da das Häufigkeitsverhältnis von Schuppen und Epithelzellen (SchE-Verhältnis) in bestimmten Gebieten des Hinterflügels unabhängig vọn der Größe der Epithelzellen konstant ungefähr $1: 8$ beträgt ( 8 -Gebiete), wurde gefolgert, daß die Schuppenbildung nicht auf einem Determinationsakt beruht, bei dem einzelne verstreut stehende Zellen innerhalb eines bis dahin homogene: Epithels als SchStZ I ausgezeichnet werden, sondern auf einer von allen Zellen der Flügelanlage als Schuppen-

2 W. K öh l e r, Z. Morph. Ökol. Tiere 24, 582 [1932]; M. S t o ß b e r g, ebenda 34, 173 [1938].

3 K. H e nk e u. G. Rön s ch, Naturwiss. 38, 335 [1951]; R. K ru m i n šs, Biol. Zbl., im Druck.
Vorderflügels mit ungefähr gleichen Häufigkeiten von Tiefen- und Mittelschuppen wurden 6-Gebiete mit Werten von etwa 1:6 gefunden, auf seiner Oberseite, wo gegenüber der Unterseite ein Teil der Mittelschuppen durch Deckschuppen ersetzt ist, 5,7-Gebiete. Diese quantitativen Verhältnisse waren mit dem einfachen Ansatz zu deuten, daß diejenigen EStZ, deren Schwesterzelle eine Mittelschuppe mit der Kernstufe $16 \mathrm{n}$ liefert, nicht drei, sondern nur zwei Teilungsschritte durchmachen, so daß jeweils vier Epithelzellen entstehen, und solche EStZ schließlich, deren Schwesterzelle eine Deckschuppe mit der Kernstufe $32 \mathrm{n}$ hervorbringt, nur einen Teilungsschritt. Da die Kernstufen der Schuppenbildungszellen (SchBiZ) auf verschiedene Anzahlen von Endomitoseschritten zurückzuführen sind, die der Tiefenschuppen auf zwei, der Mittelschuppen auf drei und der Deckschuppen auf vier, wird so dem DT-Schritt 3 eine kompensatorisch determinierende Wirkung auf die mitotische Tätigkeit der

4 A. K üh n u. K. H e n k e, Nachr. Ges. Wiss. Göttingen, math.-physik. Kl. N.F. 15 (H. 2), 127 [1932].

5 K. H e n k e, Biol. Zbl. 65, 120 [1946].

${ }_{6}$ K. Henke u. J. M ertz, Biol. Zbl. 61, 40 [1941]. 
beiden in seinem Gefolge auftretenden Zellgruppen zugeschrieben. Er soll einen für Exo- und Endomitoseschritte in gleicher Weise maßgebenden Faktor, der allen SchEStZ in gleicher Quantität zukommt, auf die beiden mit ihm gebildeten Schwesterzellen nach verschiedenen Schlüsseln verteilen und damit bestimmen, daß mit einer vergleichsweise höheren Kernstufe in der Schuppenbildungszelle eines Schuppenepithelverbandes eine kleinere Anzahl von Teilungsschritten der zugehörigen Epithelstammzelle verknüpft ist und umgekehrt. Die Summe aus der Anzahl der Endomitoseschritte, welche die Kernstufe der Schuppenbildungszelle einstellen, und der Anzahl der Exomitoseschritte der Epithelstammzelle beträgt in allen Fällen 5. Dieses in der DT 3 wirksame Kompensationsprinzip bietet ein neuartiges Problem für die Physiologie der Zellteilung.
Die neue Feststellung schließlich, daß meist zwei als a- und b-Verband unterschiedene Zweierverbände paarweise nebeneinander stehen, führt auf die Annahme des DT-Schrittes 1, der als a-b-Teilung eine Viererverbandstammzelle in eine a- und eine b-Zweierverbandstammzelle zerlegt. Aus diesen letzten gehen mit der DT 2 die vier SchE-Stammzellen ap, ad, bp und bd hervor, die gemeinsam einen Viererverband als bisher höchste noch in sich differenzierte Einheit des Schuppenkleides hervorbringen. Nach den Feststellungen von B ö d e wa d t t über die Anzahlen der Teilungsschritte auf den verschiedenen Stadien der Metamorphose ist zu folgern, daß die DT $1-3$ in der Mitosenperiode der Vorpuppe ablaufen.

Die SchE-Verhältniswerte waren bisher nur für einzelne ausgewählte Regionen der verschiedenen

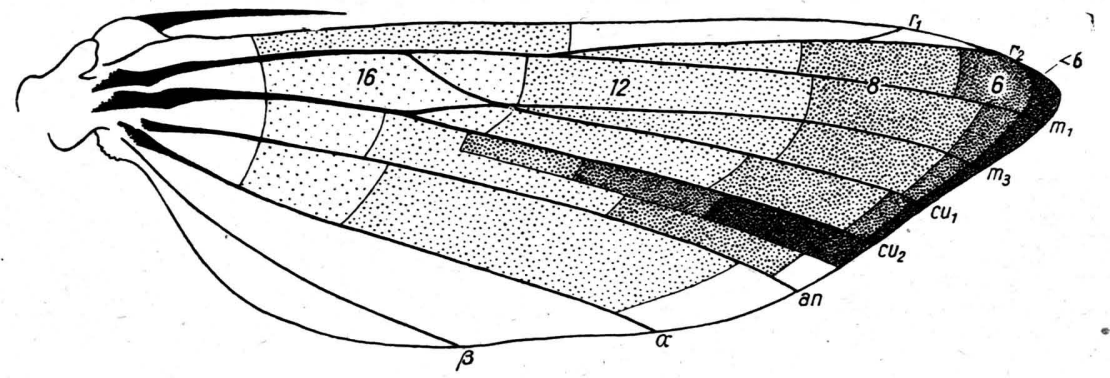

Abb. 2. Oberseite des Puppenhinterflügels mit Eintragung der Gebiete mit verschiedenen Schuppen-EpithelVerhältniswerten (Anzahlen von Epithelzellen je Schuppe):16-Gebiete, 12-, 8-, 6-, <6-Gebiete. Weiß die nicht genau geprüften Gebiete. Halbschematisch. $35 / 1$.

Der DT-Schritt 2 wurde aus dem schon erwähnten Verhältnis der Anzahlen von einerseits Tiefenschuppen, andererseits Mittel- oder Deckschuppen auf dem Vorderflügel erschlossen. Der Umstand, daß die Tiefenschuppen hier stets die Hälfte der Beschuppung ausmachen, ist begreiflich, wenn von den beiden aus dem vor der DT 3 ablaufenden Teilungsschritt hervorgehenden Zellen in jedem Fall die eine einen Tiefen-, die andere aber entweder einen Mittel- oder einen Deckschuppenepithelverband liefert. Zwei in einem entsprechenden Verhältnis zueinander stehende Sorten von SchE-Verbänden wurden jetzt auch überall am Hinterflügel festgestellt. Da aber die Polyploidiestufen der Schuppen und die Zellanzahlen in den Epithelverbänden hier meist andere sind als am Vorderflügel, müssen sie allgemeiner als bisher benannt werden. Es geschieht im folgenden nach der vorherrschenden Stellung der Vertreter der beiden Sorten zueinander, indem der in der Mehrzahl der Fälle proximal stehende Verbandtyp als p-Verband, der distal stehende als d-Ver-

- band bezeichnet wird. Je ein p- und ein d-Verband bilden zusammen einen Zweierverband. Ihre Stammzellen werden durch die DT 2 als p-d-Teilung voneinander getrennt. Insofern sich p- und d-Verbandstammzellen bei der kompensatorischen Verteilung exo- und endomitotischer Teilungspotenzen in der DT 3 verschieden verhalten, wird also mit der DT 2 jeweils die besondere Form bestimmt, in der das Kompensationsprinzip in der DT 3 wirksam wird.
Flügelflächen bestimmt worden. Neuerdings wurde nun an Totalpräparaten einer Reihe von Hinterflügeln der Puppe mit abgeschlossener Mitosenperiode teils die Ober-, teils die Unterseite in ihrer ganzen Ausdehnung systematisch aufgenommen. Das Ergebnis für die Teile der Oberseite, in denen eindeutig übersehbare Verhältnisse gefunden wurden, zeigt Abb. 2. Die Flügelfläche ist deutlich regional gegliedert. Die Darstellung ist insofern schematisiert, als die meist ziemlich schmalen Übergangszonen zwischen Gebieten mit verschiedenen SchE-Verhältniswerten nicht berücksichtigt sind, ebenso die Aderzüge, an denen die Grenzen anders verlaufen. Der in den früheren Befunden am Hinterflügel vorherrschenden SchE-Wert 8 gilt keineswegs für den ganzen Flügel, sondern nur für eine durch Verwerfungen an den Adern zerrissene Querzone. Distal von ihr liegen 6 - und $<6$-Gebiete, ähnlich wie sie früher am Vorderflügel gefunden wurden. Neu sind die 12- und 16-Gebiete in den mittleren und basalen Flügelteilen. Unter den früher von $\mathrm{He} \mathrm{n} \mathrm{k} \mathrm{e}^{5}$ in seiner $\mathrm{Abb} .5$ zusammengefaßten Befunden am Hinterflügel waren

7 G. H. B ö d e w a d t, Biol. Zbl. 70, 31 [1951]. 
Werte von 6, 12 und 16 gleichfalls mit einer gewissen Bevorzugung vertreten, doch traten sie gegenüber den um 8 liegenden stark zurück, entsprechend den damals ausgewählten, in der früheren Abb. 4 gekennzeichneten Regionen. Das neue Ergebnis zeigt, daß sie jeweils in bestimmten Gebieten gesetzmäßig auftreten.

Damit kann geprüft werden, ob sich das bisher nur aus dem summarischen Vergleich zwischen verschiedenen Flügelfächen gefolgerte Kompensationsprinzip auch für die verschiedenen Regionen einer Flügelfläche bestätigt und ob es außer bei SchE-Werten von $<6,6$ und 8 auch bei höheren gilt. Wenn das letzte zutrifft, muß in 16-Gebieten ausschließlich, in 12-Gebieten zur Hälfte neben Tiefenschuppen mit der Kernstufe 8 ein bisher nicht erfaßter, als Unter-

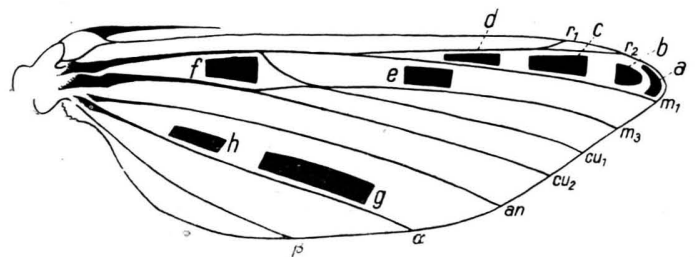

Abb. 3. Hinterflügel mit Eintragung der auf Kern- und Schuppengrößen untersuchten Flügelstellen.

tiefenschuppe zu bezeichnender Größentyp mit der Kernstufe 4 vorliegen. Das Ergebnis der Schuppenmessungen, die daraufhin vorgenommen wurden, ist mit dieser Folgerung vereinbar, doch wird der. Einklang durch weitere neu gefundene Gesetzmäßigkeiten getrübt. Es soll daher erst später betrachtet werden.

Es ist zu erwarten, daß das Kompensationsprinzip außer an den Ausmaßen der fertigen Schuppen, womöglich noch unmittelbarer, auch an den Kerngrößen der SchBi-Zellen zum Ausdruck kommt. Daher wurden an Totalpräparaten von Puppenflügeln in den in Abb. 3 bezeichneten Gebieten $a-f$ Messungen der Kerndurchmesser der SchBi-Zellen durchgeführt.

Bei den früheren Untersuchungen 6 über die Kerngrößen auf dem Flügel in Schnittpräparaten verschiedener Altersstufen wurden im Puppenalter von 108-114 Stdn. bei $25^{\circ}$ die größten Unterschiede der Kerndurchmesser gefunden. Aus der Verteilung der verschiedenen in diesem Stadium gemessenen Werte wurde für den Vergrößerungsfaktor des Kernvolumens beim Übergang von einer Kernstufe zu der nächsthöheren der Wert 2 erschlossen. Die neuen Untersuchungen an Totalpräparaten mußten aber an einem etwas früheren Stadium, bei einem Puppenalter von 84 bis 96 Stunden durchgeführt werden, da die aus- wachsenden Schuppenfortsätze das Bild später zu unübersichtlich machen.

Abb. 4 zeigt das Ergebnis. Die Kerngrößen des 8-Gebietes (Abb. $4 c$ ) bilden eine einfache Fehlerkurve. Im 6-Gebiet (Abb. $4 b$ ) ist die Variabilität vergrößert, und zwar in der Hauptsache durch eine Verschiebung eines erheblichen Teils der Varianten nach der Seite der größeren Kerne. Der Kurvenverlauf ist gut vereinbar mit der Annahme, daß neben dem im 8-Gebiet allein vorkommenden Größentyp der Tiefenschuppenkerne mit gleicher Häufigkeit ein zweiter größerer Typ erscheint, entsprechend der Ausbildung gleicher Anzahlen von Tiefen- und Mittelschuppen in diesem Gebiet. Durch Zerlegung der Variationskurve in zwei je $50 \%$ der Varianten umfassende Kurven, von denen eine der Kurve für das 8-Gebiet möglichst angeglichen ist, ist dies Ergebnis in der Kurvendarstellung veranschaulicht.

In dem $<6$-Gebiet (Abb. $4 a$ ) treten noch größere Kerne auf und es erscheint ein Nebengipfel der Kurve, welcher auf einen neuen Größentyp, die auf Kosten eines Teils der Mittelschuppenkerne gebildeten Kerne der Deckschuppen mit der Stufe 32 deutet.

Als Vergrößerungsfaktor für das Kernvolumen beim Übergang von den Tiefen- zu den Mittel- und weiter zu den Deckschuppenkernen ergibt sich hier ein Wert von ungefähr 1,6. Eine Reihe von Werten für die Kerndurchmesser mit dem entsprechenden Zunahmefaktor $=1,17$ ist in die Abbildungen als Parallelen zur Ordinate eingetragen. Daß sich hier ein niedrigerer Wert für die Kernvergrößerung bei ansteigender Polyploidiestufe als bei den früheren Messungen ergibt, ist verständlich, da jetzt ein jüngeres Stadium mit noch nicht voll ausgebildeten Größenunterschieden untersucht wurde.

Das Kompensationsprinzip, das bisher nur aus einem Vergleich der verschiedenen Vorder- und Hinterflügelflächen abgeleitet wurde, bestätigt sich also bei einem Vergleich verschiedener Teilgebiete der Hinterflügeloberseite. Dabei sind zunächst die beteiligten Sch E-Werte und Bildungskernstufen noch die gleichen wie früher.

Geht man nun von der Kerngrößenkurve des 8- zu der des 12-Gebietes über, so erscheint eine Erweiterung der Variationsbreite nach der Seite der kleineren Kerne (Abb. $4 d+e$ ). Entsprechend der Erwartung, daß in einem 12-Gebiet neben Tiefenschuppen in gleicher Anzahl wie sie Untertiefenschuppen mit der Kernstufe 4 gebildet werden, wurde auch diese Kurve in zwei gleichflächige Teilkurven zerlegt, deren eine wieder der Kurve für das 8-Gebiet möglichst 
angepaßt ist. Die Durchmesser in der Gruppe der kleineren Kerne liegen im Umkreis eines Wertes, gegenüber dem der charakteristische Wert für die Tiefenschuppenkerne um den Faktor 1,6 vergrößert ist. Der Befund stimmt also mit der Erwartung befriedigend überein.
Dięse Verschiebung kann damit zusammenhängen, $\mathrm{da} ß$ das untersuchte 16-Gebiet in einer von allen anderen ziemlich weit entfernten Flügelregion basal der Discoidalquerader liegt (Abb. 3). Es besteht die Möglichkeit, daß im basalen Flügelteil besondere, die Kerngröße bestimmende Faktoren wirksam sind. Be-
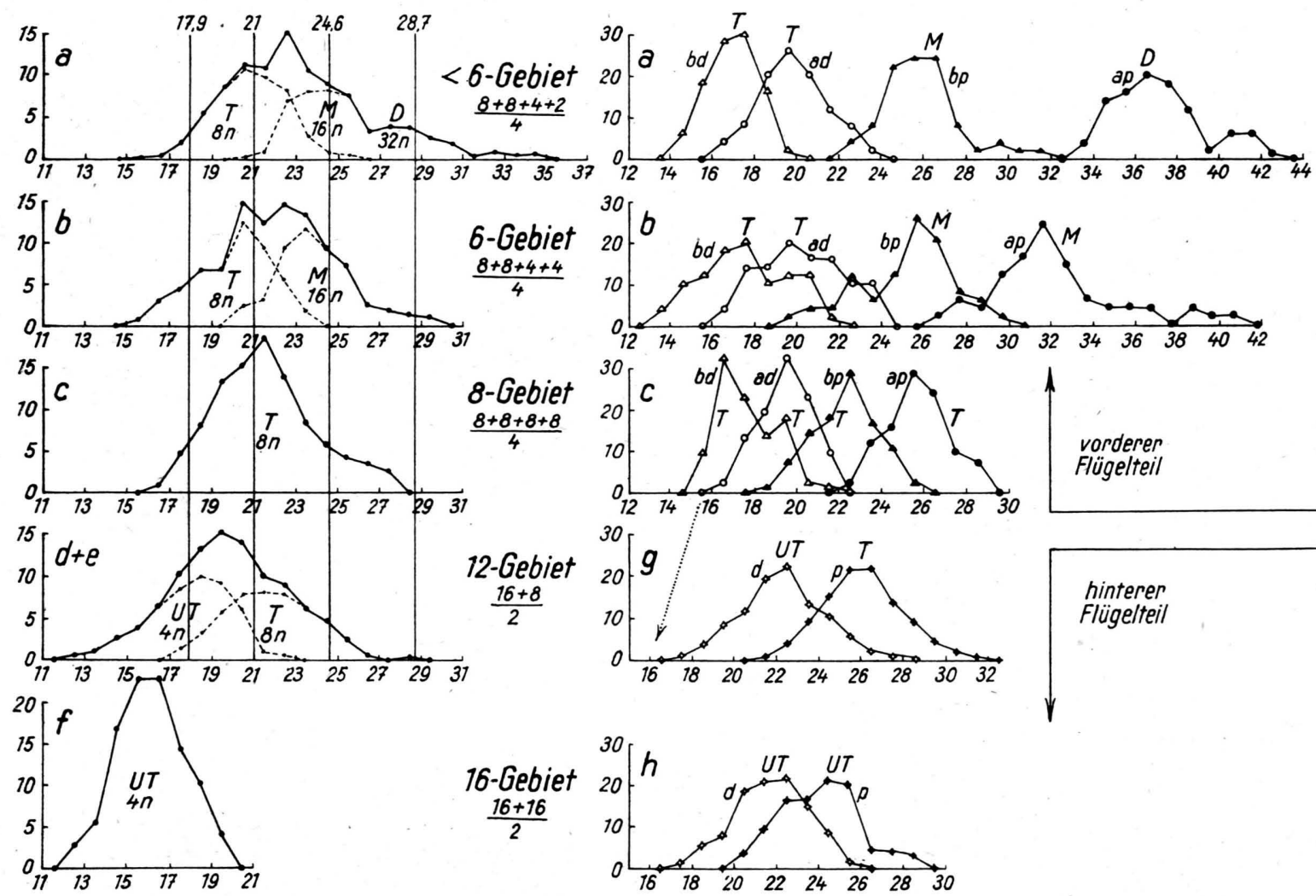

\section{hinterer
Flügelteil}

Abb. 5.

Abb. 4.

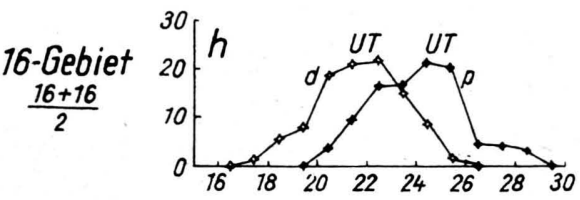

Abb. 4. Prozentkurven für die mittleren Durchmesser der Schuppenbildungskerne (Maßeinheit 1/3 $\mu$ ) im Puppenalter 84-96 Std. bei $25^{\circ}$ an den Flügelstellen $a, b, c, d+e, f(\mathrm{Abb} .3)$ mit verschiedenen Schuppen-Epithel-Verhältniswerten. Unter den Bezeichnungen der Gebiete die Deutungen ihrer Verhältniswerte als Durchschnittswerte aus den Verhältniswerten der jeweils in einem Viererverband $(a-c)$ bzw. in einem Zweierverband $(d+e, f)$ vereinigten Schuppen-Epithel-Verbände. Kurven mit verschiedenen Kerngrößentypen hypothetisch zerlegt. UT Untertiefenschuppenkerne, $T$ Tiefen-, $M$ Mittel-, $D$ Deckschuppenkerne, darunter die Polyploidiestufe. Die Parallelen zur Ordinate bezeichnen die Durchmesser einer Reihe von Kernen, deren Volum sich schrittweise um den Faktor 1,6 vergrößert. Anzahl Kerne je Befundkurve 200-400.

Abb. 5. Prozentkurven für die Länge der Schuppenkörper (Maßeinheit 4,4 $\mu$ ) bei den Viererverbandschuppentypen $a p, b p, a d, b d$ an den Flügelstellen $a, b, c$ und bei den Zweierverbandtypen $p$ und $d$ an den Stellen $g$ und $h$

(Abb. 3). UT, T, M, D Bezeichnung nach der Kernstufe (Abb. 4), Anzahl Schuppen je Flügelstelle rund 200.

Die Kurve $f$ für die Kerngrößen eines 16-Gebietes schließlich zeigt entsprechend der Annahme, daß hier nur ein Kerngrößentyp vorliegt, geringe Streuung, liegt aber entgegen der Erwartung bei niedrigeren Werten als die aus dem Befund für die 12-Gebiete abgeleitete Kurve für die Untertiefenschuppenkerne. schränkt man sich zunächst auf das 12-Gebiet, so wird jedenfalls hier die durch Extrapolation aus den Ergebnissen für Gebiete mit niederen Sch E-Werten gewonnene Voraussage quantitativ bestätigt. Das Kompensationsprinzip gilt also auch für höhere Sch EWerte als bisher bekannt war und führte zur Auf- 
findung eines neuen, auf seiner Grundlage vorausgesagten Kerngrößentyps.

Bekanntlich bieten die Schmetterlinge bei zytologischen Untersuchungen besonders an den somatischen Kernen erhebliche Schwierigkeiten. Immerhin war es möglich, auf Grund der Ausbildung von Chromo-
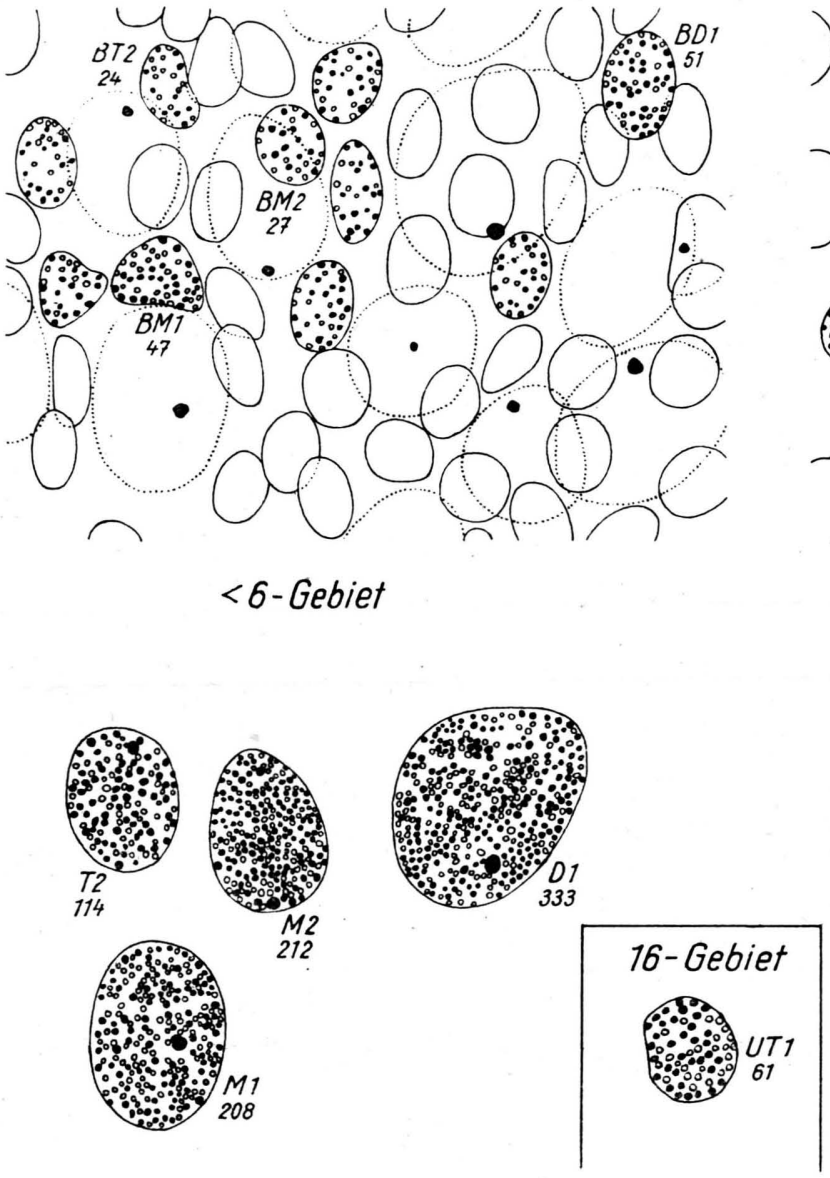

F e u lg en gefärbt und mit Lichtgrün gegengefärbt waren, zeigten in allen Ruhekernen mehr oder weniger deutlich getrennte Chromozentren in mit der Kerngröße steigender Anzahl. Eine Zählung ist schon bei den Epithelkernen mit der kleinsten Anzahl immer nur bei einzelnen Kernindividuen möglich, stets recht schwierig und mühsam und nicht immer ganz gleich reproduzierbar. Unmöglich ist sie überall da, wo die vielfach über-

Abb. 6. Kernumrisse aus dem Oberseitenepithel des Hinterflügels der Puppe (84-96 Std., 25 ${ }^{\circ}$ ), teilweise mit Eintragung der Chromozentren. Grundepithelkerne unbezeichnet. Schuppenbildungskerne: UT von Untertiefenschuppen, $T$ von Tiefen-, $M$ von Mittelschuppen, $D$ Deckschuppenkern; für $<6$ - und 12-Gebiet unten aus den oberen Übersichtsbildern isoliert. Balgbildungskerne mit $B$ und der Bezeichnung des zugehörigen Schuppenbildungskerns bezeichnet. Unter den Kernbezeichnungen die Anzahlen der gezählten Chromozentren. $<6$-Gebiet nahe der Flügelstelle $a$ (Abb. 3) $r 2$ genähert; 12-Gebiet Stelle $d$ nahe $m 1$; 16-Gebiet Stelle $f$. Totalpräparat. Fixierung: Bouin-Allen. Färbung: Feulgen Lichtgrün. 2000/1 (gezeichnet 4000/1).

zentren in den Ruhekernen die Polyploidiestufe einzelner Kerne verschiedener Größentypen im Epithel mit einiger Sicherheit direkt zu bestimmen und damit die aus den Kernmessungen gezogenen Schlüsse zu bestätigen.

Untersucht wurden wieder die Hinterflügel von Puppen, die bei $25^{\circ}$ ein Alter von 84 bis 96 Stdn. erreicht hatten. Totalpräparate, die nach $\mathrm{B}$ ou in-Allen fixiert, nach einander liegenden Kerne nicht einwandfrei optisch zu isolieren sind. Zwar stört das Unterseitenepithel des Flügels bei Betrachtung der Oberseite nur ausnahmsweise, dagegen können Epithel- und auch Balgkerne den tiefer liegenden Schuppenbildungskernen der gleichen Seite sehr dicht aufliegen.

Die Zählungen wurden an Hand von Kernzeichnungen mit Eintragung der ungefähren Orte der einzeln ansprechbaren Chromozentren ausgeführt (Abb. 6). Die 


\begin{tabular}{|c|c|c|c|c|c|c|c|c|c|c|c|c|}
\hline & $U T_{1}$ & $U T_{2}$ & $U T_{3}$ & $T_{1}$ & $T_{2}$ & $T_{3}$ & $T$ & $M$ & $M_{1}$ & $M_{2}$ & $M$ & $D_{1}$ \\
\hline Zählung & 61 & 62 & & 112 & 114 & 118 & 125 & 194 & 208 & 212 & 270 & 333 \\
\hline Schätzung & & 65 & & 114 & 127 & 119 & & & 229 & 224 & 274 & 379 \\
\hline Mittlerer Durchmesser & 14,6 & 17,6 & 18,0 & 20,4 & 19,5 & 20,0 & & & 24,6 & 21,3 & & 30,4 \\
\hline
\end{tabular}

Tab. 1. Gezählte und geschätzte Anzahlen der Chromozentren und mittlere Durchmesser (Maßeinheit ${ }^{1 / 3} \mu$, vgl. Abb. 4) für verschiedene Schuppenbildungskerne. Die bezifferten Kerne sind in Abb. 6 eingezeichnet. UT Untertiefen-, $T$ Tiefen-, $M$ Mittel-, $D$ Deckschuppen.

Zeichnungen geben nur die Kernumrisse richtig wieder, nicht das wirkliche Bild des Kerninhaltes. Die Chromozentren sind durchaus nicht immer so deutlich getrennt, wie sie in den Zeichnungen erscheinen, und ihre unterschiedliche Größe wurde im allgemeinen nicht zum Ausdruck gebracht. Nur ein einzelner feulgenpositiver Körper von besonderer Größe, der wenigstens bei den größeren Kernen recht regelmäßig zu identifizieren ist und als einzelnes Chromozentrum gezählt wurde, ist lagerichtig eingetragen. Die Figuren stellen also nicht mehr als eine Vorarbeit für eine erste Art von Zählungen dar, bei der die Anzahl der Chromozentren möglichst erst bei einer Übertragung der gezeichneten Orte auf eine Pause durch Abzählen bestimmt wurde. Bei den größeren, selbst in günstigen Fällen auf den ersten Blick ganz unanalysierbar erscheinenden Kernen wurde außerdem noch ein anderes Verfahren angewandt, das kurz als Schätzung gegenüber den an Hand von Punktzeichnungen vorgenommenen Zählungen bezeichnet werden soll. Hier wurden entsprechend dem bei einer bestimmten mittleren Einstellung auftretenden Bild des Kernes mit unregelmäßig verteilten dunkleren und helleren Zonen je nach Kerngröße etwa 5 bis 10 Teilgebiete festgelegt und im Umriß skizziert. Darauf wurden die einzelnen bei mittlerer Einstellung stets wieder erkennbaren Teilgebiete mit wechselnder Einstellung ausgezählt, die Teilanzahlen notiert, und zuletzt wurde die Gesamtsumme errechnet. Beide Verfahren sind außerordentlich roh und fehlerbelastet, müssen aber auch bei den großen Kernen wenigstens einen Anhalt für die Größenordnung der Chromozentrenanzahlen geben.

Die Zählung der Chromozentren an 10 Kernen des Grundepithels, von denen 9 in Abb. 6 eingetragen sind, ergab folgende Werte:

$$
25,26,27,28,29,30,30,31,32,40 \text {. }
$$

Der Durchschnitt beträgt 29,8, bei Ausschluß des letzten Extremwertes 28,7, liegt also jedenfalls in der Nähe von 30 .

Wagne $r^{8}$ stellte bei seiner Untersuchung der Gametogenese von Ephestia haploid $29+1$ Chromosomen fest. $\mathrm{Da}$ die Epithelkerne mindestens diploid sein müssen, könnte man zunächst daran denken, daß nur ungefähr die

9 H. O. W a g n e r, Z. Zellforschg. mikroskop. Anat. 12. 749 [1931].
Hälfte der Chromosomen im Ruhekern durch Chromozentren gekennzeichnet ist. Auffallend ist es indessen, daß $\mathrm{K} \ddot{\mathrm{hhle}} \stackrel{2}{2}$ bei gelegentlichen, nach der Untersuchung von Wagner auf späten Entwicklungsstadien vorgenommenen Zählungen an Prophasen und frühen Metaphasen somatischer Mitosen gleichfalls nur 27-30 Chromosomen fand (l. c. S. 628). Die Angabe bedarf dringend der Nachprüfung. Sollte sie bestätigt werden, so wäre zu prüfen, ob etwa in der Furchung eine Elimination von Chromosomen stattfindet. Zur Deutung der Befunde am Ruhekern ist schließlich auch an die Möglichkeit einer scmatischen Paarung der Homologen zu denken, die freilich bisher nur für Dipteren bekannt ist. Eine weitere Frage ist es dann, ob etwa auch das Bild der Mitose durch somatische Paarung bestimmt wird und sich so der Befund von $\mathrm{K}$ ö h l e r erklärt.

Die Ergebnisse der Zählung und Schätzung der Anzahlen der Chromozentren in einigen Schuppenbildungskernen zeigt Tab. 1.

Einzelne einigermaßen auszählbar scheinende Kerne wurden zunächst, unter Berücksichtigung des Größenverhältnisses zu den Nachbarkernen und des Verhältnisses von Größe und Chromozentrendichte, einer bestimmten Kernstufe zugeordnet, dann gezeichnet und die Chromozentrenanzahl gezählt und geschätzt. Im allgemeinen ist es recht wohl möglich, in einem bestimmten Flügelgebiet mit verschiedenen Kernstufen die einzelnen Individuen anzusprechen. Die in die Tabelle aufgenommenen Angaben über die an den Zeichnungen gemessenen mittleren Durchmesser ermöglichen es, sich an Hand der Abb. 4 ein Bild von der Stellung der einzelnen Kernindividuen innerhalb der Variationsbreite der Größe des betreffenden Kerntyps in der jeweils untersuchten Flügelregion zu machen.

Die Werte für die Anzahlen der Chromozentren schwanken, besonders in der Gruppe der als Mittelschuppenkerne angesprochenen Kernindividuen, sehr stark und sind, wie erwähnt, im allgemeinen nur näherungsweise reproduzierbar. Die Schätzungswerte liegen durchweg höher als die Zählwerte. Eine Überschneidung zwischen den für Vertreter verschiedener Stufen gefundenen Anzahlen kommt aber nirgends vor, sehr im Unterschied zu der stark transgredierenden Variation der Kerngrößen. Die Durchschnitts- 
werte aller Angaben für die einzelnen Stufen der Schuppenbildungskerne ergeben die Reihe

$\begin{array}{cccc}\text { UT-Kerne } & T \text {-Kerne } & M \text {-Kerne } & D \text {-Kerne } \\ 63 & 118 & 230 & 356\end{array}$

Bis zu dem Wert für die Mittelschuppenkerne ergibt sich das klare Bild einer Verdoppelungsreihe mit einer Grundanzahl von etwa 30. Der auffallend niedrige Wert für den Deckschuppenkern, der kaum

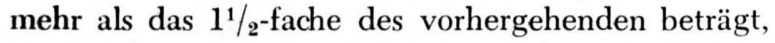
kann an der besonderen Schwierigkeit der Untersuchung der extrém großen Kerne liegen. Vielleicht ist aber auch der Prozeß der Vermehrung der Chromozentren hier noch nicht abgeschlossen. Im ganzen ist jedenfalls der Schluß berechtigt, daß die Kerngrößentypen tatsächlich auf einer Polyploidie verschiedenen Grades beruhen, so wie es in ihrer Bezeichnung nach Kernstufen zum Ausdruck gebracht wurde. Die in ihrer Größe sehr verschiedenen, als Mittelschuppenkerne angesprochenen Individuen $M 1$ und M 2 enthalten ähnliche Anzahlen von Chromozentren und bestätigen damit ihre Beurteilung nach dem Augenschein. Besonders wichtig ist es, daß der Kern UT 1 aus einem 16-Gebiet (Abb.6) mit seinen 61 Chromozentren eindeutig der Kernstufe 4 mit der doppelten Grundanzahl zugehört. Die Verschiebung der Kerngrößenkurve für das 16-Gebiet in Abb. $4 f$ gegenüber der konstruierten Teilkurve für die Untertiefenschuppenkerne in dem untersuchten 12-Gebiet hängt also nicht mit der Polyploidiestufe zusammen. Sie muß auf besonderen, regional in verschiedener Weise auf das Kernvolum wirkenden Faktoren beruhen. Die extrapolierende Anwendung des Kompensationsprinzips auf Gebiete mit Sch E-Werten von mehr als 8 gelingt also nicht nur für 12-, sondern auch für 16-Gebiete.

Untersuchungen an den noch nicht geklärten hinteren Teilen des Hinterflügels sind im Gang. Hier scheinen, wie es nach dem Kompensationsprinzip möglich ist, starke Unterschiede der Kernstufe mit mittleren Sch-E-Werten kombiniert zu sein. Am Flügelrand mit seinen sehr großen Randfransenkernen stößt die Zählung der Epithelzellen auf Schwierigkeiten. Besondere Verhältnisse liegen sicher bei der von 20 bis 30 riesigen Bildungszellen gemeinsam aufgebauten Haftborste am Vorderrand der Hinterflügelbasis vor (vgl. Abb. 2). Die Bildungszellkerne enthalten hier so viele Chromozentren, daß sicher mehr als 5 Endomitoseschritte durchgemacht werden müssen.

Dem niedersten Kerngrößentyp mit der Stufe 2n gehört außer den Grundepithelkernen nach den früheren Untersuchungen ${ }^{6}$ über das Kernvolum auch ein Teil der Balgbildungszellkerne an. Daneben erscheint unter den Balgkernen ein zweiter größerer Typ, dem die Kernstufe 4 zugeschrieben wurde. Er ist von dem ersten nur sehr. unvollkommen abgesetzt. Später" wurde gezeigt, daß diesen zwei Kernstufen zwei Längentypen der Schuppenbälge zugeordnet werden können. Beide Balglängentypen können bei Tiefen-, Mittel- und Deckschuppen auftreten. Im allgemeinen kommen sie bei den Mittelschuppen mit vergleich. barer Häufigkeit vor, während bei den Tiefenschuppen die kurzen, bei den Deckschuppen die langen Bälge überwiegen. Die beiden Balgkerntypen lassen sich nun auch nach den Anzahlen der Chromozentrer eindeutig unterscheiden.

Im allgemeinen liegen die Balgbildungskerne etw in der Höhe der Epithelkerne oder wenig tiefer, deutlich höher als die zugeordneten Schuppenbildungskerne und ihnen gegenüber in der Auswachsrichtung der Schuppen, d. i. distal oder in der Richtung zum Flügelrand verschoben. Die Ausschnitte Abb. 6 wurden so gewählt, daß sie eine den typischer. Fall repräsentierende Zusammenstellung von auszählbaren Schuppen- und Balgbildungszellkernen enthalten. Der dem Tiefenschuppenkern T2 mit einiger Sicherheit zuzuordnende Balgkern BT 2 zeigt, wie aus Abb. 6 ersichtlich, 24 Chromozentren, gehört also der Grundstufe 2 der Kerne an. Zu dem Deckschuppenkern D 1 gehört wahrscheinlich der im Bild? stark nach rechts, in der Richtung zum nahen Flügelrand verlagerte große Kern $B D 1$ mit seinen ir. Abb. 6 eingetragenen, der Kernstufe 4 entsprechenden 51 Chromozentren. Von den Balgkernen der Mittelschuppen $M 1$ und $M 2$ wurden für den einen 47. für den anderen 27 Chromozentren gezählt. Offenbar handelt es sich im ersten Fall um den Kern eines langen Balges mit der Stufe 4, im zweiten um einen kurzen Balg mit der Kernstufe 2. In der Umgebung der Untertiefenschuppenkerne fanden sich im allgemeinen nur kleine Kerne mit etwa 30 Chromozentren. Die Bälge der Untertiefenschuppen dürften danach allein oder ganz überwiegend dem kurzen Typus angehören. Die beiden aus dem Kernvolum und den fertigen Bälgen erschlossenen Balgkernstufen kommen also auch in den Chromozentrenanzahlen zum Ausdruck. In ihrer Kernstufe stimmen die kurzen Bälge mit den Epithelzellen, die langen mit den Untertiefenschuppen überein.

An einer Stelle des in Abb. 6 dargestellten 12-Gebietes lag ein kleiner Kern völlig innerhalb des Um:

9 K. H e n k e, Nachr. Akad. Wiss. Göttingen, math.physik. Kl. 1945, 20. 
risses eines größeren, als Untertiefenschuppenkern angesprochenen Kerns, und zwar so dicht auf ihm, $\mathrm{da} ß$ er nur an dem Schatten erkennbar war, welcher in seinem Bereich bei Tiefeinstellung unter die Ebene der benachbarten Epithelkerne ebenso wie an den Orten dieser letzten erschien. Anscheinend handelte es sich um den zugehörigen Balgkern (Abb. 6, UT 3 + BUT 3). Die Auszählung des nicht zerlegbaren Komplexes ergab 85 Chromozentren. Der Fall erscheint als Bewährungsprobe für die Mö̀glichkeit, Kernstufen nach den Anzahlen der Chromozentren zu bestimmen.

Überblickt man die Schuppengröße am Totalpräparat des fertigen Hinterflügels, so bemerkt man beim Übergang von den nur Tiefenschuppen führenden 8-Gebieten über die 6- zu den $<6$-Gebieten deutlich das Hinzutreten der Mittel- und zuletzt der Deckschuppen. Dagegen zeichnet sich beim Fortgang von den 8- zu den 12- und 16-Gebieten der Ersatz der Tiefen- durch Untertiefenschuppen kaum ab. Betrachtet man andererseits einzelne Flügelstellen genauer, so bemerkt man, daß außer den mit den Kernstufen verknüpften Größenunterschieden noch weitere vorhanden sind, die zwar mit jenen in Beziehung stehen, aber auch innerhalb der 8- und 16-Gebiete mit jeweils nur einer Kernstufe erscheinen.

Unter den Tiefenschuppen der 8-Gebiete lassen sich deutlich vier in mehr oder weniger regelmäßigem Wechsel angeordnete, ungefähr gleich häufige Typen unterscheiden. Daraufhin wurde ein zusammenhängendes Gebiet bei mehreren Flügeln systematisch in Viererverbände aufgeteilt, wobei stets nur vereinzelte nicht einzuordnende Schuppen übrigblieben. Die Länge der einzelnen Schuppenkörper wurde gemessen und die Werte wurden in vier Gruppen registriert, wobei die Zuordnung jeder Schuppe zu einer Gruppe nach ihrem vorher bestimmten Typ innerhalb ihres Verbandes vorgenommen wurde. Die Kurvendarstellung Abb. $5 \mathrm{c}$ zeigt vier sich stark überschneidende Fehlerkurven. Ihre Zusammenfassung würde eine viergipfelige Kurve ergeben und diese Form bleibt praktisch unverändert erhalten, wenn man die Werte für die nicht eingeordneten Schuppen hinzufügt. Unter den Tiefenschuppen sind also vier ungefähr gleich häufige Längentypen vorhanden. Zwei Typen, der bei Ordnung nach abnehmender Länge an dritter oder an vierter Stelle stehende, ähneln in ihrer Stellung auf dem Flügel Tiefenschuppen, die beiden anderen Mittel- und Deckschuppen der 6- und $<6-\mathrm{Ge}-$ biete. Da nun die Schuppen in den letztgenannten
Gebieten mehr oder weniger deutlich in Zickzack- bis Doppelreihen stehen, in denen die größeren Schuppen bevorzugt die proximalen, die Tiefenschuppen die distalen Plätze einnehmen, werden jetzt allgemein die Schuppen des 1. und 2. Typus als p-Schuppen, die des 3. und 4. als d-Schuppen bezeichnet. Das paarweise Auftreten von $p$ - und d-Schuppen wurde schon früher auf eine gemeinsame Zweierverbandstammzelle und ihre Teilung durch einen jetzt als DT 2 bezeichneten differentiellen Teilungsschritt zurückgeführt (Abb. 1). Soweit nun nach den neuen Befunden die Zweierverbände ihrerseits in ungleichen Paaren auftreten können, müssen auch noch Viererverbandstammzellen angenommen werden, welche jeweils durch einen dem DT-Schritt 2 noch vorausgehenden differentiellen Teilungsschritt, den DT-Schritt 1, in die beiden Zweierverbandstammzellen $a$ und $b$ zerlegt werden. Nach der Stellung der Schuppen auf dem Flügel ist es wahrscheinlich, daß immer eine pSchuppe vom 1. und eine d-Schuppe vom 3. Längentyp aus der einen, andererseits eine p-Schuppe vom 2. und eine d-Schuppe vom 4. Längentyp aus der anderen Art von Zweierverbandstammzellen hervorgehen. Hiernach wurden die vier Typen in Abb. $5 \mathrm{c}$ als ap, ad, bp und bd bezeichnet. Es ist aber nicht ganz ausgeschlossen, daß in Wahrheit die Schuppen des 1. und 4. sowie andererseits die des 2. und 3. Längentypus aus Schwesterzellen hervorgehen.

Unter den Schuppen des 6-Gebietes sind die entsprechenden vier Typen leicht wiederzufinden (Abb. 5 b). Während ad und bd ihren Charakter als Tiefenschuppen mit der Kernstufe $8 \mathrm{n}$ behalten, sind ap und bp entsprechend ihrem Übergang in Mittelschuppen mit der Kernstufe $16 \mathrm{n}$ erheblich länger geworden. Geht man weiter zu den Flügelgebieten mit Sch E-Werten von $<6$ über, so lassen sich hier einzelne Ausschnitte finden, in denen regelmäßig die drei kürzesten Typen eines Viererverbandes sich so wie im 6-Gebiet verhalten, während ap, offenbar nun als Deckschuppe mit der Kernstufe 32, wiederum erheblich an Länge zugenommen hat (Abb. $5 \mathrm{a}$ ). In solchen Gebieten müßte, wenn sie an Präparaten von Puppenflügeln eindeutig $\mathrm{zu}$ erkennen wären, ein Sch E-Wert von $(8+8+4+2) / 4=5,5$ gefunden werden. Sie sind aber nur am fertig beschuppten Flügel einigermaßen klar von den größeren 6-Gebieten abzugrenzen. Der früher für den Vorderflügel gefundene Wert 5,7 ist offenbar so zu deuten, daß die ap-Schuppen in einem Teil der erfaßten Viererverbände ebenso wie alle bp-Schuppen Mittelschuppen, in den übrigen aber Deckschuppen darstellen. 
Die bisher nach den vorkommenden Schuppenlängen betrachteten 8-, 6- und <6-Gebiete liegen verhältnismäßig nahe beieinander, in der Hinterflügelspitze. Der Fortgang von den 8- zu den 12- und 16-Gebieten, in denen die Untertiefenschuppen mit der Kernstufe $4 \mathrm{n} \mathrm{zu}$ suchen sind, wird durch den Umstand erschwert, daß die Größe einer Schuppe nicht nur von der Kernstufe und von der auch bei gleicher Kernstufe im Zusammenhang mit den DT 1 und 2 auftretenden Größendifferenzierung abhängt, sondern außerdem noch, relativ unabhängis hiervon, von ihrem Ort innerhalb der Gesamtflügelfläche. Am Hinterflügel nimmt die Schuppengröße u. a. besonders in Richtung von vorn nach hinten zu. Bei einem Übergang von dem näher untersuchten 8-Gebiet $c$ zu den 12-Gebieten $d$ und $e$ sowie dem 16-Gebiet $f$ (Abb. 3) würde dieser Faktor weniger stören, doch sind die Schuppen in $f$ oft deformiert und daher auch ihre Ausmaße schwer zu untersuchen. Aus diesem Grund wurden die untereinander gut vergleichbaren Gebiete $g$ und $h$ gewählt. Die Messungsergebnisse sind in Abb. 5 eingetragen. Zum Ausgleich für die mit dem Übergang vom vorderen zum hinteren Flügelteil verknüpfte Verschiebung der Meßwerte sind die Teilfiguren $g$ und $h$ gegenüber den vorhergehenden gleichmäßig so weit nach links verschoben, daß die Kurven in $g$ im Vergleich zu $c$ ungefähr die gleiche Lage einnehmen wie die hier nicht wiedergegebenen Messungsergebnisse für die Gebiete $d$ und $e$ im vorderen Flügelteil.

Eine a-b-Differenzierung der Schuppen ist ebenso wie in anderen 12- und 16-Gebieten weder nach dem Augenschein noch durch Messungen festzustellen. Wohl aber sind in beiden Gebieten die mit ungefähr gleicher Häufigkeit vorhandenen p- und d-Typen anzusprechen. Die beiden bei der Messung getrennt registrierten Typen des 12-Gebietes (Abb. $5 \mathrm{~g}$ ) ergeben zwei sich überschneidende einfache Fehlerkurven mit unterschiedlicher Gipfellage. Die des p-Typus liegt bei höheren Maßen. Sie ist als Tiefenschuppenkurve aufzufassen, entsprechend den beiden Kurven für ap und bp in Abb. $5 c$. Die d-Kurve in Abb. $5 \mathrm{~g}$ muß die nach der Untersuchung der Kernvolumina und der Chromozentrenbilder in den Gebieten $d$ und $e$ zu erwartenden Untertiefenschuppen umfassen. Ihr Schwerpunkt liegt trotz der bei der Zusammenstellung der Kurven vorgenommenen groben Korrektur keineswegs links, sondern eher rechts von dem der beiden Kurven für ad und bd in Abb. $5 c$ zusammengenommen. Der Unterschied von Tiefen- zu Untertiefenschuppen kommt also in der hier allein betrachteten Schuppenlänge nicht zum Ausdruck. Geht man nun zu dem ebenso wie $g$ im hinteren Flügelteil gelegenen 16-Gebiet $h$ über, so findet man den Gipfel der Kurve der d-Schuppen ungefähr an der gleichen Stelle wie im 12-Gebiet, während die p-Kurve deutlich zu niederen Werten verschoben und damit der
d-Kurve angenähert ist. Dies Verhältnis entspricht der Erwartung, denn im 16-Gebiet sind nur Untertiefenschuppen anzunehmen, unter denen allein durch die pd-Differenzierung bedingte Längenunterschiede auftreten sollen, während im 12-Gebiet der mit der höheren Kernstufe des als Tiefenschuppe ausgebildeten p-Typus verknüpfte Unterschied hinzutritt.

Mit der Erfassung der a-b- und p-d-Differenzierung wird die offenbar sehr empfindliche Variabilität der Schuppengröße um einen Schritt weiter als bisher erfaßt. Die Analyse wurde dadurch erschwert, $\mathrm{da} \beta$ in den 12- und 6-Gebieten die $\mathrm{p}$-d-, in den $<6$-Gebieten außerdem die a-b-Differenzierung mit dem Auftreten verschiedener Kernstufen einhergeht, die bisher - neben dem auf dem Hinterflügel jedenfalls nicht so wie auf dem Vorderflügel wirksamen Farbmuster - als einziger Faktor der Schuppengröße bekannt waren. Dabei handelt es sich nun nicht um eine Überlagerung voneinander unabhängiger Faktoren, sondern die Schuppengröße erscheint zunächst gleichsam als Schrittmacher der Kernstufe. Geht man von dem 16- über das 12- zum 8-Gebiet, so erscheint die höhere Kernstufe zuerst in den schon am Ausgang längeren $\mathrm{p}$-, danach erst bei den d-Schuppen. Geht man weiter von dem 8- zum 6-Gebiet, so erhalten allein die längeren p-Schuppen die höhere Kernstufe, und schließlich ist es beim Fortgang zum $<6$-Gebiet noch einmal der schon vorher längste ap-Typ, welcher zu der erhöhten Kernstufe übergeht. Die mit den DT 1 und 2 gesetzten Zelldeterminationen wirken sich also in jedem Fall an der Zellgröße aus, in bestimmten Fällen, offenbar bei der Überschreitung bestimmter Schwellen, außerdem, und zwar in gleichem Sinn, an der Kernstufe. Hieraus ist wohl zu schließen, daß die Kernstufe von einem nicht unmittelbar auf sie, sondern primär auf die Zellgröße wirkenden Faktor abhängt. Andererseits wirkt aber offenbar auch die Kernstufe ihrerseits auf die Zellgröße, denn sonst wären beispielsweise die sprunghaften Größenänderungen der ap-Schuppen bei dem Übergang von dem 8- zum 6- und weiter zum 5,5-Gebiet in den Kurven Abb. 5 nicht zu verstehen. Diese Sprünge beruhen nicht etwa darauf, daß in einer größeren Zone mit gleitender Größenzunahme des ap-Typus einzelne durch nichterfaßte Zwischenräume getrennte Gebiete ausgewählt wurden. Wird der ganze Raum einschließlich der Zwischenzonen gleichmäßig aufgenommen, so ergeben die ap-Typen allein eine deutlich dreigipfelige Kurve, wie es dem Vorkommen von Tiefen-, Mittelund Deckschuppen allgemein entspricht. Wenn also 
die Kerngröße nicht ihrerseits auf die Schuppengröße wirkte, müßte entweder der unmittelbar die Größe der ap-Schuppen einstellende Faktor sich beim Übergang von einem Flügelgebiet zum anderen ähnlich wie bei einem Farbstreifenmuster diskontinuierlich verändern, oder die Schuppengröße müßte auf einen sich gleichförmig verändernden Faktor umschlagend reagieren. Das letzte ist in sich unwahrscheinlich, das erste deshalb, weil nicht einzusehen ist, warum die Folge von Zonen sprungweise zunehmender Wirkungsstärke des fraglichen Faktors gerade so gestuft sein sollte, daß sie die Bildungszellkerne zu einer in gleichem Schritt gehenden Erhöhung ihrer Polyploidiestufe veranlassen könnte. Auch die Größenvariabilität der bei experimentell ausgelöster Regeneration des Epithels auftretenden Riesenschuppen ${ }^{10}$, welche eine auf rhythmische Verdoppelung des Zellvolumens deutende Diskontinuität aufweist ${ }^{11}$, ist wohl nur aus einer Abhängigkeit der Schuppengröße von der Kernstufe zu verstehen. Die primär mit den DT 1 und 2 auftretende Größendetermination der Schuppen, die sich in den 8- und 16-Gebieten allein auswirkt, kann als plasmatisch der in bestimmten Fällen in Abhängigkeit von ihr auftretenden sekundären, über die Kernstufe wirkenden Determination als einer genomatischen gegenübergestellt werden. Die erste dürfte ihrer Natur nach- eine gleitende Größenzunahme bewirken können, denn die Größendifferenzierung der ap-, bp-, ad- und bd-Schuppen nimmt anscheinend innerhalb des 8-Gebietes mit dem Fortgang nach dem Außenrand des Flügels hin zu. Die zweite hingegen wirkt ihrer Natur nach diskontinuierlich und klinkt gewissermaßen die Schuppengröße entsprechend den Kernstufen auf bestimmte bevorzugte Variationsbereiche ein. Diese Gesetzmäßigkeit dürfte für die Wachstumsbeziehungen von Zelleib und Kern von allgemeiner Bedeutung sein.

Dagegen stellt das Kompensationsprinzip wohl einen Spezialfall dar. Immer wenn die mit den DT 1 und 2 verknüpfte Größendetermination der Schuppenbildungszelle bestimmte Grade erreicht, wird von dem in der Sch E-Stammzelle in konstanter Menge vorhandenen Teilungsfaktor ein weiterer Anteil auf Kosten der Epithel- der Schuppenstammzelle zugeteilt. Dies geschieht bei der DT 3, der Teilung der Sch EStammzelle, wirkt sich aber erst zwei Zellgenerationen später an der Schuppenbildungszelle und, wie früher ${ }^{6}$ zeigt und oben S. 72 durch Bestimmung der Chromozentrenanzahlen bestätigt wurde, in gleichem

10 A. K üh n u. H. P i e p h o, Biol. Zbl. 60, 1 [1940].

11 A. K ü h n, Z. Naturforschg. 4 b, 104 [1949].
Sinn, aber nicht in gleichem Ausmaß an der Balgbildungszelle aus. Soweit die mit der DT 3 nach dem Kompensationsprinzip geschaffene Situation nicht nachträglich noch geändert werden kann, wird die von der Schuppe zu erreichende Kernstufe und mit ihr die Anzahl der zugeordneten Epithelzellen schon bei der Bestimmung der Verlaufsweise der DT 3 durch die DT 1 und 2 festgelegt.

Außer durch die Untersuchungen am Normalflügel wurde das differentielle Teilungsgeschehen bei der Metamorphose der Mehlmotte an Hand von Durchstrahlungsversuchen studiert. Beginnend mit der Zeit

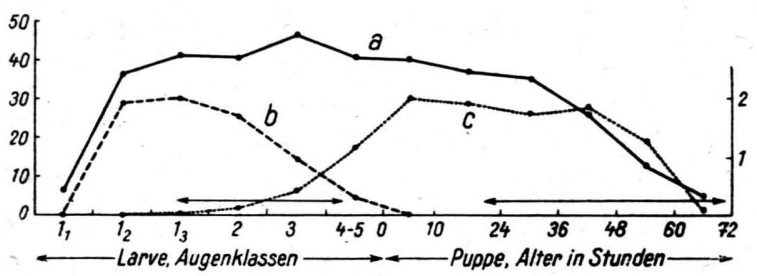

Abb. 7. Ergebnisse der Röntgendurchstrahlung von Larven des letzten Stadiums und von Puppen auf verschiedenen Altersstufen. $a$ Prozentuale Verminderung der Anzahl der Schuppenapparate. $b$ Prozentuale Verkleinerung der Flügelfläche. $c$ Prozentuale Häufigkeit deformierter Bälge ohne Schuppe unter den nach Bestrahlung vorhandenen Schuppenapparaten. Altersangaben für die Larven in Augenklassen nach $\mathrm{K} \ddot{\mathrm{u} h \mathrm{n}}$ und $\mathrm{Pi}$ e pho ${ }^{12}$. Die Pfeile über der Abszisse markieren die Mitosenperioden der Vorpuppen- und der Puppenzeit. - Untersuchte Flügelregion zwischen $c u 1$ und $c u 2$, - für $c$ außerdem zwischen $r 2$ und $m 1$ (Abb. 3). Ordinatenteilung für $a$ und $b$ links, für $c$ rechts aufgetragen. Röntgenlicht: $180 \mathrm{kV}$, spezifische Dosis für $a$ und $b 4000 \mathrm{r}$, für c $3000 \mathrm{r}$.

nach der letzten Larvenhäutung wurden Raupen, Vorpuppen und junge Puppen in einer fortlaufenden Reihe aufeinander folgender Altersstufen mit Röntgenlicht $(180 \mathrm{kV})$ behandelt. Die überlebenden Tiere zeigten nach der Metamorphose verschiedene Veränderungen des beschuppten Epithels. Hier sollen zunächst drei von diesen Veränderungen hauptsächlich insoweit erörtert werden, als sie gemeinsam den besonderen Charakter des DT-Schrittes 3 zum Ausdruck bringen.

In Abb. 7 gibt die Kurve $a$ eine Verminderung der Anzahl der gebildeten Schuppenapparate nach Behandlung verschiedener Altersklassen wieder, Kurve $b$ eine Verkleinerung der Flügelfläche.' Da die Schuppen auf dem verkleinerten Flügel nicht merklich zusammengedrängt, sondern in ähnlicher Ordnung wie auf dem normalen Flügel stehen, muß die Flächenverkleinerung mindestens in ihrem überwiegenden Teil auf einer Verringerung nicht der Größe, sondern der 
Anzahl der Grundepithelzellen beruhen. Kurve c schließlich stellt den Ausfall von Schuppen bei Deformierung der Bälge dar.

Die Kurven $a$ und $b$ steigen im Anfangsteil des letzten Larvenstadiums gleichermaßen an und verweilen bis in den Beginn der durch einen Pfeil markierten Mitosenperiode der Vorpuppenzeit hinein gemeinsam auf einem ähnlichen Niveau von etwa 30 bis $40 \%$. Dies Verhalten läßt auf die Ausschaltung eines Teils der Schuppenepithelstammzellen und ihrer Vorfahren infolge der Strahlenwirkung schlieBen. Jeder Zellausfall hat den Verlust eines oder mehrerer Schuppenapparate samt den zugeordneten Epithelverbänden zur Folge. Der nun folgende Abfall der Kurve $b$ für Epithelzellenausfall bei anhaltendem Verlust von Schuppenapparaten ist als Ausdruck der DT 3 zu deuten, die nach B ö d e w a d t ${ }^{7}$ gegen Ende der Vorpuppenmitosenperiode stattfindet. Durch diesen Teilungsschritt werden die Schuppenepithelstammzellen in Schuppenstammzellen I und Epithelstammzellen zerlegt. Diese letzten sind samt ihren Abkömmlingen weitgehend strahlungsresistent, während ihre Schwesterzellen, wie der weitere horizontale Verlauf der Kurve $a$ anzeigt, über die Zeit der Verpuppung hinweg zunächst praktisch unverändert empfindlich bleiben. Schließlich, während der in der Abbildung gleichfalls markierten Mitosenperiode der Puppenzeit, fällt auch Kurve $a$ ab. Hier kommt zum Ausdruck, daß mit der Zerlegung der Schuppenstammzellen in Bildungszellen eine Ausschaltung ganzer Schuppenapparate nicht mehr möglich ist.

Das Auftreten der Strahlenempfindlichkeit der Flügelzellen nach der letzten Raupenhäutung scheint mit der Vorbereitung der für die Metamorphose des Epithels charakteristischen Schuppenbildung zusammenzuhängen. Bekanntlich wird der Impuls zur Metamorphose auf hormonalem Wege erteilt 12, 13. Die ersten äußerlich sichtbaren Anzeichen für das Einsetzen seiner Wirksamkeit sind Veränderungen an der Hautfarbe und den Augen der erwachsenen Raupe. Ungefähr bis zu diesem Zeitpunkt ist es möglich, die Metamorphose auf experimentellem Wege, durch Einpflanzung überzähliger Corpora allata, aufzuschieben. An Stelle der bevorstehenden Puppenhäutung tritt dann eine überzählige Raupenhäutung ein. Spezielle Untersuchungen mit parallel durchgeführten Transplantations- und Durchstrahlungsversuchen müßten wohl zur Klärung der Beziehungen zwischen den bekannten hormonalen Faktoren der Metamorphose und der in den Durchstrahlungsversuchen erfaßten Vorbereitung des Epithels für die Schuppenbildung beitragen.

Kurve $c$ für den Ausfall von Schuppen bei Deformierung der Bälge ist einer anderen Versuchsreihe

12 A. K ühn u. H. Pi e pho, Nachr. Ges. Wiss. Göttingen VI (Biol.) 2 (Nr. 9), 141 [1936]. als $a$ und $b$ mit geringerer spezifischer Dosis entnommen und daher nur hinsichtlich der Verlaufsweise, nicht in der Höhe der auftretenden Werte mit ihner vergleichbar. Ihr Anstieg beginnt während der Vorpuppenmitosenperiode gleichzeitig mit dem Absinker der Kurve $b$, und zur Zeit der Verpuppung, während Kurve $b$ auf 0 fällt, erreicht sie ihr Maximum. Der Abfall der Kurve $b$ spiegelt, wie oben gezeigt, den DT-Schritt 3 in dem Auftreten der Epithelstammzellen wieder. In dem gleichzeitigen Anstieg der Kurve wird nun offenbar derselbe Prozeß noch einmal erfaßt, diesmal in der zunehmenden Häufigkeit der Schuppenstammzellen I. Neben der schon bei ihren Vorfahren möglichen, zur Ausschaltung ganzer Schuppenapparate führenden Strahlenwirkung können diese Zellen noch eine weitere aufnehmen, welche nur die Schuppe zum Verschwinden bringt, nicht aber den Balg. Im Fortgang der Puppenmitosenperiode erlischt die Reaktion. Sie kann also nicht unmittelbar an den Schuppen- und Balgbildungszellen ausgelöst werden, wenigstens nicht sobald sie einen gewisser: Differenzierungsgrad erreicht haben, wohl aber durch eine Beeinflussung der vorhergehenden beiden Zellgenerationen, der Schuppenstammzellen I und II. Wie der Ausfall der Schuppe und die Deformierung des Balges im einzelnen zustandkommt, muß noch näher untersucht werden. Insbesondere ist zu fragen. inwieweit sich dabei etwa die an Hand anderer Beziehungen früher ${ }^{6},{ }^{9}$ festgestellte Entwicklungsabhängigkeit zwischen Schuppen- und Balgbildungszelle auswirkt.

Vielfach erscheinen als Folge der Strahlenwirkuns mannigfach veränderte Schuppen. Einzelne abgeänderte Schuppentypen treten nach Durchstrahlung verschiedener Entwicklungsstadien immer in der gleichen Form auf, und zwar bei Behandlung junger Stadien in größeren, später in kleineren Nestern, schließlich nur noch einzeln stehende Schuppen. Im Unterschied zu den von $\mathrm{K} \ddot{\mathrm{uhn}}{ }^{14}$ durch Hitzereizung jeweils nur in bestimmten sensiblen Perioden erzielten Modifikationen handelt es sich hier offenbar um somatische Mutationen, und jeder zusammenhängende Komplex sowie jede einzeln stehende mutante Schuppe ist auf ein Mutationsereignis zurückzuführen. Eine besonders häufige derartige Mutation betrifft die Form und besonders die Farbe des Schuppenkörper, und wird als dunkle Schuppen bezeichnet. Da sie nur

13 H. P i e p h o, Biol. Zbl. 60, 367 [1940]; derselbe. Biol. Zbl. 65, 141 [1946].

14 A. K ü h n, Biol. Zbl. 61, 109 [1941]. 
bei Weibchen beobachtet wird, ist sie als geschlechtskontrolliert oder, bêi der Heterogametie der Weibchen, als rezessiv geschlechtsgebunden anzusehen. Näher untersucht wurde bisher nur das Auftreten dieser Mutation auf den Flügeln von Tieren, die nach der letzten Raupenhäutung behandelt waren. Es ergaben sich unerwartete Befunde, die gewisse Folgerungen hinsichtlich der Teilungsvorgänge in der Entwicklung des beschuppten Epithels zulassen.

Die mittlere Nestgröße, bestimmt als durchschnittliche Anzahl der in einer Gruppe vereinigten mutanten Schuppen, hat bei der hier verwendeten Strahlenart und Dosis für die jüngsten nach der letzten Raupenhäutung behandelten Tiere einen Wert von etwa 5 (Abb. 8, Kurve $a$ ). Bei Behandlung älterer Tiere fällt die Kurve rasch ab. Zur Zeit der einsetzenden Mitosenperiode, bei Augenklasse $1_{3}$, beträgt der Wert nur noch etwa 1,7 , in Klasse 2, vor dem Maximum der Zellteilungstätigkeit, 1,2. Jetzt erscheint also im Gefolge einer Mutation in der überwiegenden Mehrzahl der Fälle nur noch eine einzelne abgewandelte Schuppe. Bald danach, entsprechend dem Wert 1,0, werden Gruppen von abgeänderten überhaupt nicht mehr gebildet, sondern es treten nur noch einzeln stehende mutante Schuppen auf, und zwar nach Durchstrahlungen in dem ganzen Zeitraum bis zur Ausdifferenzierung der Schuppen bei den 60 bis 72 Stunden alten Puppen.

Úberraschend ist es zunächst, daß nach Strahlenwirkung schon vor dem Einsetzen der Zellteilungstätigkeit überwiegend einzeln stehende mutante Schuppen ausgelöst werden können. Für den Vorderflügel ist aus Untersuchungen über die Zellenanzahlen in der Anlage auf verschiedenen Entwicklungsstadien $^{7}$ bekannt, daß während der Mitosenperiode der Vorpuppenzeit aus jeder vorher vorhandenen Zelle durchschnittlich 8 Schuppenstammzellen hervorgehen, und nach den Größenverhältnissen der Anlagen muß für den Hinterflügel ähnliches gelten. Die vor der Mitosenperiode ausgelösten Mutationen müssen also in vielen Fällen so beschaffen sein, daß sie nur bei einem Bruchteil der Schuppen in Erscheinung treten, welche aus der Zelle, in der die Mutation eintrat, hervorgehen.

Weiterhin besagt der erhebliche Abfall der Kurve für die mittlere Nestgröße in dem Zeitraum vor Einsetzen der Mitosenperiode, daß die Anzahlen der aus einer Zelle hervorgehenden mutanten Schuppen in

16 Ch. A u e r b a c h, Publ. Stazione Zool. Napoli 22, Suppl., 1 [1950]; M. V o i g t, Z. Vererbungslehre 83, 324 [1950]; di e s e l b e, ebenda 83, 341 [1950]. dieser Zeit abnimmt. Die in diesem Zeitraum ausgelösten unerwartet geringen Nestgrößen können also nicht lediglich auf einer Subletalwirkung oder unvollständigen Penetranz beruhen, da nicht einzusehen ist, warum die Anzahl der absterbenden oder der die Mutation nicht manifestierenden Schuppen mit zunehmendem Alter der Zelle, in der die Mutation entstand, abnehmen sollte. Vielmehr muß in den Zellen der Flügelanlage eine in der Zeit zwischen letzter Raupenhäutung und dem Einsetzen der Zellteilungstätigkeit zunehmende Anzahl selbständig mutationsfähiger Einheiten vorhanden sein, die später, während der Mitosenperiode, auf ihre Abkömmlinge ver-

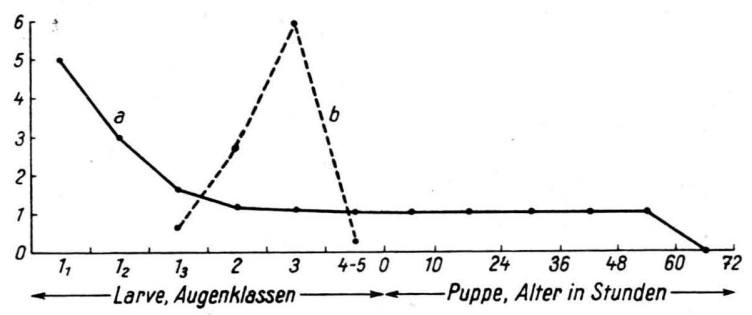

Abb. 8. $a$ Durchschnittliche Anzahlen der im Anschluß an einen Mutationsvorgang entstandenen mutanten dunklen Schuppen (= durchschnittliche Mutantennestgrößen) nach Röntgenbehandlung verschiedener Altersstufen. Untersuchte Flügelregion vorwärts Ader $a$ (Abb.3). $b$ Durchschnittliche Anzahlen der Mitosen in der Hinterflügelanlage nach den Angaben von Querner15 zur Kennzeichnung der Mitosenperiode in der Vorpuppe. Altersangaben für Larven wie in Abb. 7. Ordinateneinheit für $a$ 1 Schuppe, für $b 100$ Mitosen.

teilt werden. Nun handelt es sich offenbar um einen chromosomalen Faktor, und man kann bei den Zellen der Flügelanlagen mit einem Aufbau der Chromosomen aus einer Mehrzahl struktureller Einheiten in der Art von Chromonemen rechnen, die eine mit der Zeit bis zum Einsetzen der Zellteilungen zunehmende Anzahl jeweils unabhängig voneinander, aber in sich einheitlich mutierender Gruppen bilden. Die Folgerung ist im Einklang mit Ergebnissen anderer genetischer ${ }^{16}$ und zytologischer ${ }^{17}$ Untersuchungen über chemisch oder durch Strahlen induzierte Mutationen. In dem vorliegenden Fall ist es möglich, das zeitliche Fortschreiten der Aussonderung von Mutationseinheiten innerhalb der einzelnen Zelle zu verfolgen. Sie geschieht hier offenbar in Vorbereitung der zeitlich abgesetzt rasch aufeinander folgenden Zellteilungen. Wenn diese letzten beginnen, ist der Prozeß in der

17 F. Oehlkers u. H. Marquardt, Z. Vererbungslehre 83, 299 [1950]; H. M a rquard t, Naturwiss. 37, 416, 433 [1950]; B. M. S li z y n s k i, Genetics 35, 279 [1950].

15 H. Q u e rne r, Biol. Zbl. 67, 293 [1948]. 
überwiegenden Mehrzahl der Zellen mindestens so weit fortgeschritten, daß für jede der später mit dem DT-Schritt 2 auftretenden Sch E St Z eine isoliert mutationsfähige Einheit vorhanden ist, die, wenn sie mutiert, später eine Einzelschuppe markiert.

Wann der Vorgang beginnt, läßt sich bisher nicht angeben, da die Nestgrößenkurve von dem ersten festgelegten Punkt an abfällt. Es ist möglich, daß er ähnlich wie das Auftreten der besonderen, zu einer Ausschaltung von Stammzellen durch die Behandlung führenden Empfindlichkeit, das in dem Anstieg der Kurven $a$ und $b$ in Abb. 7 hervortritt, mit der Bestimmung des Epithels zur Metamorphose zusamenhängt. Die Vermehrung der chromosomalen Substanz, die mit der Vermehrung der Zellen nötig wird, kann entweder auch schon in der Vorbereitungszeit der Mitosenperiode, bei der Vermehrung der Mutationseinheiten stattfinden, oder später im Zuge der Aufteilung dieser letzten durch die Zellteilungen im Lauf der Mitosenperiode selbst.

Die Markierung von Schuppen gemeinsamer Herkunft durch somatische Mutationen gibt ein Mittel an die Hand, die Wachstumsordnung der Flügelanlage, soweit sie durch Zellteilungen bedingt ist, $\mathrm{zu}$ studieren. Versuche hierzu mit Durchstrahlung verschiedener Raupenstadien sind im Gang. Bei den hier geschilderten Untersuchungen bestand zunächst die Erwartung, daß es möglich sein würde, auch die $\mathrm{Ab}$ folge der vor der DT 3 auf dem letzten Raupenstadium vorhandenen Stammzellen und den wenigstens zum Teil differentiellen Charakter ihrer Teilungen, der bisher nur aus der Zusammensetzung der fertigen Beschuppung aus den a-b- und p-d-Schuppentypen erschlossen wurde, an Hand der Größe und Zusammensetzung der zu verschiedenen Zeiten nach d:r let:ten Raupenhäutung ausgelösten Mutantennester zu erfassen. Da nun aber schon vor der Mitosenperiode eine Vermehrung der selbständigen Mutationseinheiten in den Zellen der Flügelanlage eintritt, ist mit dieser Möglichkeit nicht mehr ohne weiteres zu rechnen.

Stammzellen, bei denen die mutationsfähige Anlage als ganze mutiert, werden in jedem Fall eine durch die Mutation einheitlich markierte Deszendenz hervorbringen. Wenn aber eine Teilmutation eintritt und danach die Vermehrung der Einheiten vor Beginn der Zellteilungen noch fortschreitet, können zwei Möglichkeiten unterschieden werden. Im ersten Fall geschieht die Aufteilung der Einheiten während der Mitosenperiode in derselben Abfolge, in der sie entstanden sind, geordnet, im zweiten mehr oder weniger zufallsmäßig, ungeordnet. Im ersten Fall werden die Abkömmlinge eines mutierten Elementes schließ- lich allein und vollzählig in eine Stammzelle eingehen und die Nachkommenschaft diesër letzten in einem Mutantennest zur Anschauung bringen. Nun ist anzunehmen, daß die Vermehrung der Mutationseinheiten in jeder Zelle der Flügelanlage und entsprechend auch die spätere Vermehrung der Zelle selbst einheitlich fortschreitet, so daß die Gliedanzahl in den Verbänden, die jeweils aus einer Zelle hervorgehen, sich nur in Zweierpotenzen bewegt. Damit sind aber, neben einzeln stehenden mutanten Schuppen, auch nur Mutantennester solcher Größen zu erwarten. Ferner werden die einzelnen Nester in ihrer Zusammensetzung aus den verschiedenen a-b- und p-d-Typen den differentiellen Charakter der DT 1 und 2 zur Darstellung bringen.

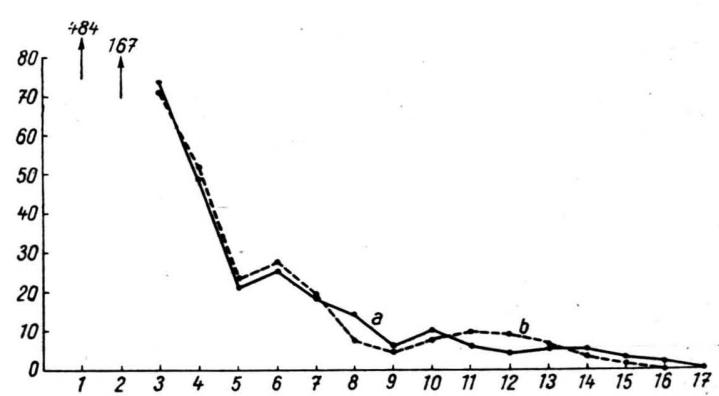

Abb. 9. $a$ Beobachtete absolute Anzahlen von Mutantennestern verschiedener Größe ( $1-17$ Schuppen je Nest) nach Röntgenbehandlung im letzten Larvenstadium, Augenklasse 1 bis 2 (Abb. 8). $b$ Erwartung für die gleiche Gesamtanzahl von Nestern wie in der Befundkurve unter der Annahme, daß die Nester unvollständige Verbände darstellen, weil nur $70 \%$ der mutanten Schuppen erfaßt werden.

Eine Betrachtung des gesamten während des letzten Raupenstadiums auf den Stufen der Augenklassen 1 und 2 behandelten Materials zeigt aber, daß beides nicht zutrifft. Vielmehr kommen alle Nestgrößen von 1 bis 16 vor (Abb. 9, Kurve $a$ ), ferner Nester von der Größe einer Zweierpotenz, die in ihrer Zusammensetzung nicht der Erwartung entsprechen, beispielsweise Zweiergruppen aus zwei p- oder zwei d-Schuppen, Vierergruppen mit drei p-Schuppen und so fort.

Das letzte ist erklärlich, wenn nicht die soeben erörterte erste, sondern die zweite Möglichkeit verwirklicht ist, nach der die bei Beginn der Mitosenperiode in den einzelnen Stammzellen vorhandenen Einheiten im Vergleich zu ihrer Entstehungsfolge ungeordnet verteilt werden, weil nun, beispielsweise statt eines geschlossenen Viererverbandes, vier vom Zufall bestimmte Schuppen aus zwei von Schwesterzellen gebil- 
deten Viererverbänden markiert sind. Andererseits sind auch von 1 und der Reihe der Zweierpoténzen abweichende Nestgrößen zu erwarten, wenn die den Zellteilungen vorauseilende Bildung zufallsmäßig verteilter Einheiten nicht nur bis zur Stufe der Sch E St Z, sondern noch einen oder mehrere Schritte weitergeht. Mutierte Einheiten, die bei geordneter Verteilung Schuppen markiert hätten, werden dann in Epithel- und gegebenenfalls auch in nicht zu Schuppen werdenden Zellen der Schuppenapparate, die bei geordneter Verteilung ein nicht mutiertes Element erhalten hätten, für die Beobachtung gleichsam versteckt werden, und umgekehrt werden Schuppen, die bei geordneter Verteilung normal geblieben wären, eine Markierung erhalten. So werden Nestgrößen entstehen, dire teils über, teils unter der bei geordneter Verteilung auftretenden liegen.

Mutantennester, die von der zunächst gehegten Erwartung in der Größe oder auch nur in der Typenzusammensetzung abweichen, können aber auch bei geordneter Verteilung der vor der Mitosenperiode gebildeten chromosomalen Einheiten entstehen, und zwar dann, wenn die Mutation subletal wirkt oder sich nicht in allen Fällen manifestiert, so daß unvollständige mutante Verbände erscheinen. Eine solche Beschaffenheit der Anlage würde zwar, wie oben S. 77 gezeigt, den Abfall der Nestgrößenkurve in Abb. 8 vor Beginn der Mitosenperiode nicht erklären, kann aber natürlich trotzdem bestehen und für die zunächst unerwarteten Nestgrößen und -zusammensetzungen verantwortlich sein. Die Erwartungen, die sich hier ergeben, sind für Reihen mit verschiedenen Häufigkeiten der einzelnen bei Zweierpotenzen liegenden Verbandgrößen und für verschiedene Erfassungswahrscheinlichkeiten der Mutanten leicht zu berechnen. Als Beispiel soll hier nur die Größe der Mutantennester bei der Summe der auf den Stadien der Augenklassen 1 und 2 behandelten Individuen betrachtet werden. Bei der Annahme, daß jede Schuppe eines von einer einzelnen vollmutierten Stammzelle gebildeten Verbandes mit der konstanten Wahrscheinlichkeit von 70\% zur Beobachtung kommt, ergibt sich die der Befundkurve $a$ möglichst angepaßte Erwartungskurve $b$ in Abb. 9. Berücksichtigt man die bei den höheren Nestgrößen noch ziemlich geringe Anzahl der Varianten, so kann die Anpassung wohl als befriedigend gelten. Insbesondere die charakteristischen Anstiege der Befundkurve von Nestgröße 5 nach 6 sowie von 9 nach 10 ließen sich in der Erwartung nachbilden. Die Variationsreihe der Nestgrößen ist also auch mit der Annahme einer geordneten Verteilung der chromosomalen Einheiten vereinbar, wenn die Mutation subletal wirkt oder unvollständig penetrant ist. Auch die zunächst unerwarteten Typenzusammensetzungen der Mutantennester widersprechen der Annahme wenigstens für die qualitative Betrachtung nicht. Für eine quantitative Behandlung reicht das Material noch nicht aus.

Von den beiden der Konstruktion der Erwartungskurve zugrunde gelegten Möglichkeiten, Subletalität oder unvollständige Penetranz der Mutation dunkle Schuppen, ist die zweite sehr unwahrscheinlich, da sie bei großen Mutantennestern, wie sie nach Raupenbehandlung bereits mehrfach erhalten wurden, zur Bildung von $30 \%$ in die Nester eingesprengter normaler Schuppen führen müßte. Die Erscheinung könnte selbst bei den wenigen bisher vorliegenden Fällen nicht wohl der Beobachtung entgehen. $\mathrm{Ob}$ die Deutung der Befunde mit der Annahme einer Subletalwirkung der Mutation das richtige trifft, soll zunächst durch die Untersuchung weiterer Mutationen geprüft werden, für die bisher noch kein ausreichendes Material vorlag. Wenn die Verteilung der Nestgrößen in allen Fällen dieselbe ist, wird auch diese Deutung unwahrscheinlich, da nicht damit zu rechnen ist, daß eine Reihe verschiedener Mutationen stets den gleichen Grad von Subletalität aufweist. Es bliebe dann wohl nur noch die vorher betrachtete Möglichkeit, daß die Bildung selbständiger chromosomaler Einheiten in den Flügelzellen zu Beginn des letzten Raupenstadiums noch über die Stufe der Schuppenepithelstammzellen hinausgeht, und daß diese Einheiten in der anschließenden Mitosenperiode nicht nach der Abfolge ihrer Entstehung, sondern mehr oder weniger zufallsmäßig verteilt werden. Das Maß der Abweichung der Verteilung der Nestgrößen von der erwarteten in Zweierpotenzen hängt dann davon ab, wie weit die Aufteilung der Mutationseinheiten in den Einzelzellen der Flügelanlage geht und wie stark ihre Verteilung von der geordneten abweicht. 